\title{
Polyphenols: potential source of drugs for the treatment of ischaemic heart disease
}

Guanhua Du ${ }^{1 *}$, Lan $\operatorname{Sun}^{1}$, Rui Zhao ${ }^{1}$, Lida Du ${ }^{2}$, Junke Song ${ }^{1}$, Li Zhang ${ }^{1}$, Guorong He ${ }^{1}$, Yongxiang Zhang ${ }^{3}$, Juntian Zhang ${ }^{1}$

1. Beijing Key Laboratory of drug target research and drug screening, State Key Laboratory for Bioactive Substances and Functions of Natural Medicines, Institute of Materia Medica, Chinese Academy of Medical Science and Peking Union Medical College, Beijing 100050, China.

2. School of Biomedical Sciences, The Chinese University of Hong Kong, Hong Kong, China.

3. State Key Laboratory of Toxicology and Medical Countermeasures, Beijing Institute of Pharmacology and Toxicology, Beijing 100850, China.

*: Author to whom correspondence should be addressed; E-Mail: dugh@imm.ac.cn;

Tel./Fax: +86-10-6316-5184.

\begin{abstract}
Polyphenols, which are naturally present in plants, have been studied for their chemical and pharmacological properties. Polyphenols have been found to exhibit various bioactivities such as antioxidant, free radical scavenging and anti-inflammatory effects, in addition to regulating the intracellular free calcium levels. These bioactivities are related to the underlying mechanisms of ischaemic heart diseases. Pharmacological studies have proven polyphenols to be effective in treating cardiovascular diseases in various ways, particularly ischaemic heart diseases. Based on their mode of action, we propose that some polyphenols can be developed as drugs to treat ischaemic heart diseases. For this purpose, a strategy to evaluate the therapeutic value of drugs for ischaemic heart diseases is needed. Despite several advances in percutaneous coronary intervention (PCI), the incidence of myocardial infarction and deaths due to cardiovascular diseases has not decreased markedly in China. Due to their pleiotropic properties and structural diversity, polyphenols have been of great interest in pharmacology. In the present review, we summarize the
\end{abstract}


pharmacological effects and mechanisms of polyphenols reported after 2000, and we analyse the benefits or druggability of these compounds for ischaemic heart diseases.

\section{Abbreviations}

ACEI: angiotensin-converting enzyme inhibitor;

ADMA: asymmetric dimethylarginine;

ADP: adenosine diphosphate;

ADR: adenosine receptor;

AMPK: adenosine monophosphate-activated protein kinase;

CAD: coronary artery disease;

cGMP: cyclic guanosine monophosphate;

COPD: chronic obstructive pulmonary disease;

COX: cyclooxygenase;

EC: endothelial cell;

EGCG: epigallocatechin gallate;

ER: oestrogen receptor;

FAO: fatty acid oxidation;

GPx1: glutathione peroxidase 1;

HUVECs: human umbilical vein endothelial cells;

IHD: ischaemic heart disease;

LPO: lipid peroxidation;

NADPH: nicotinamide adenine dinucleotide phosphate;

NF-кB: nuclear factor kappa B;

NO: nitric oxide; PDEs: phosphodiesterases;

OPR: opioid receptor;

PA: protocatechuic acid;

PCI: percutaneous coronary intervention;

PGI2: prostacyclin I2;

PI3K: phosphoinositide 3-kinase;

PKA: protein kinase A;

SalA: salvianolic acid A;

SalB: salvianolic acid B;

SIRT1: deacetylase sirtuin 1;

SOD: superoxide dismutase;

SP-1: specificity protein-1;

TXA2: thromboxane A2;

Keywords: Ischaemic heart diseases, Polyphenols, Flavonoids. 


\section{Contents}

1. Introduction

2. Polyphenols

3. Cardiovascular activities of polyphenols

3.1 Decrease in heart oxygen consumption or increase in oxygen supply

3.2 Anti-atherosclerotic and protect coronary vascular activities

3.3 Protective effect on myocardial cells and cardiac remodelling

3.4 Enhancing myocardial metabolism

3.5 Pro-angiogenesis and cardiocyte regeneration

4. Potential molecules for treatment of IHD

5. Conclusion and future perspective

References 


\section{Introduction}

Ischaemic heart disease (IHD) is considered the leading cause of death and disability worldwide. It is characterized by insufficient blood supply to the heart due to the pathological narrowing of coronary vessels, typical of atherosclerosis. The clinical characteristics of IHD include temporary pain (angina), irregular heartbeat (arrhythmia), permanent heart muscle damage (myocardial infarction) and loss of muscle activity (heart failure).

IHD is managed with medication and surgery. The following drugs are used to manage IHD: 1) nitroglycerine, which decreases oxygen demand and increases oxygen supply by causing the coronary artery to dilate; 2) statins, which reduce cholesterol level, improve vascular endothelial function, dilate blood vessels and inhibit inflammatory reaction; 3) angiotensin-converting enzyme (ACE) inhibitors, which reduce angiotensin II production by inhibiting ACE, increase myocardial blood flow, reduce myocardial oxygen consumption, inhibit inflammatory responses and may lower the risk of recurrent myocardial infarction; 4) calcium channel blockers, which exert cardioprotective effects by lowering the intracellular calcium level, relaxing vascular smooth muscle and dilating the blood vessels; 5) $\beta$-receptor antagonists, which reduce myocardial oxygen consumption by suppressing the effects of adrenaline; 6) anti-platelet agents, such as aspirin and clopidogrel, to inhibit platelet aggregation and lower the risk of atheromatous plaque formation; and 7) celland protein-based therapies, which help restore organ function and myocardial regeneration.

At present, percutaneous coronary intervention (PCI) with drug-eluting stents (DESs) is used to reduce cardiovascular events in patients with coronary artery disease (CAD) and IHD. Although it significantly reduced restenosis rates, the use of DES increased the risk of both late stent thrombosis and atherosclerosis, and in turn late stent failure. Many patients, especially the elderly, are deemed unsuitable for revascularization due to co-morbid diseases, unsuited anatomy or the risks inherent to the procedure (Bonetti, Holmes, Lerman, \& Barsness, 2003). Moreover, despite several advances in PCI, the incidence of myocardial infarction and deaths due to cardiovascular disease has not decreased visibly in China (Sui, Chen, \& Wang, 2015). Therefore, pharmacological interventions for IHD have been of great interest recently.

Polyphenols constitute a large group of natural products widely distributed 
across the plant kingdom. Based on the number of phenol rings and the binding structural properties, polyphenols are divided into several subclasses such as phenolic acids, flavonoids, stilbenes, lignans and tannins. In the past decades, several different polyphenols have been isolated and identified from plants, in addition to their pharmacological activities (Goszcz, et al., 2015). The evidence points to the multiple

pharmacological effects of polyphenols against cardiovascular diseases, particularly IHD. For IHD, the underlying mechanisms of action of polyphenols have been elucidated. These include reducing myocardial oxygen consumption and/or increasing oxygen supply, enhancing myocardium metabolism after ischaemia/reperfusion (I/R), inhibiting platelet aggregation and thrombosis, regulating lipid metabolism and inflammation to inhibit the formation of atheromatous plaques, promoting angiogenesis or endothelial cell (EC) repair, protecting the remaining myocardial cells and restoring myocardial contraction (Harasym \& Oledzki, 2014; Raj, Zieroth, \& Netticadan, 2015).

For this review, we systematically searched the EMBASE, PubMed and Cochrane Library databases, and the references of relevant papers. Combined with the results obtained in our laboratory, we presented an overview of the advances in elucidating the effects of polyphenols (including their derivatives and analogues) against IHD. In the following sections, we classify their pharmacological activity and functional mechanisms, particularly those with therapeutic potential for IHD.

\section{Polyphenols}

\subsection{Flavonoids}

Flavonoids are an important class of polyphenols abundantly found in vegetables, fruits and different plants; they are usually yellow in colour. Approximately $2 \%$ of the total carbon in a plant is ultimately converted to flavonoids or similar compounds. Chrysin (Figure 1A) was the first flavonoid to be isolated (1814). To date, $>4000$ flavonoids have been isolated and identified (Iwashina, 2000). Flavonoids such as quercetin (Figure 1B), rutin (Figure 1C) and hesperidin (Figure 1D), isolated from fruits, are most commonly studied for their cardiovascular effects. Furthermore, anthocyanins are common dietary flavonoids that help prevent cardiovascular disease. Although many flavonoids have been isolated, several more flavonoids are yet to be discovered.

The basic skeleton of flavonoids is composed of $\mathrm{C}_{6}-\mathrm{C}_{3}-\mathrm{C}_{6}$, generally containing 
two aromatic rings, a central three-carbon chain and a 4-carbonyl group. Flavonoids are widely used as antioxidants as they possess several phenolic hydroxyl groups that donate active hydrogen atoms and stop/delay the automatic oxidation of lipids. Many flavonoids have been found to exhibit various pharmacological actions except antioxidation. For example, pinocembrin (Figure 1E), isolated from honey and propolis, is an anti-inflammatory and antimicrobial agent that acts on diastolic arteries (Estevinho, et al. 2008; Li, et al., 2013; Rasul, et al., 2013). Baicalein (Figure 1F), isolated from the roots of the labiate Scutellaria baicalensis, can scavenge oxygen-derived free radicals and protect the heart, cerebral vessels and neurons (Xin, et al., 2014). It is worth noting that Veregen contains a mixture of known active compounds, including catechins (Figure 1K). On 31 October 2006, the Food and Drug Administration (FDA) approved the use of Veregen for treating perianal and genital condyloma (Hara, 2011). 
A<smiles>O=c1cc(-c2ccccc2)oc2cc(O)cc(O)c12</smiles>

$$
\text { chrysin }
$$

Chemical Formula: $\mathrm{C}_{15} \mathrm{H}_{10} \mathrm{O}_{4}$

C

Molecular Weight: $\mathbf{2 5 4 . 2 4}$

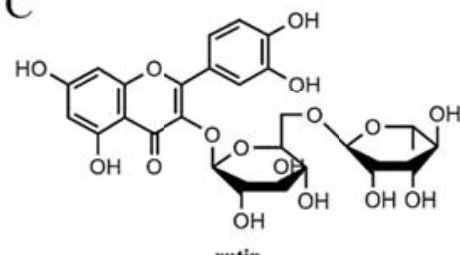

rutin

Chemical Formula: $\mathrm{C}_{27} \mathrm{H}_{30} \mathrm{O}_{16}$

Molecular Weight: 610.52

E

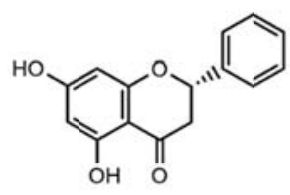

$$
\text { pinocembrin }
$$

Chemical Formula: $\mathrm{C}_{15} \mathrm{H}_{12} \mathrm{O}_{4}$

Molecular Weight: 256.26

G

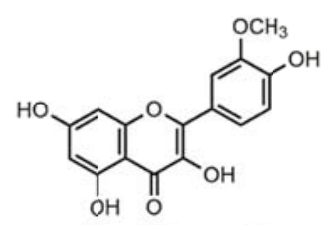

3'-0-methyl-quercetin

Chemical Formula: $\mathrm{C}_{16} \mathrm{H}_{12} \mathrm{O}_{7}$

Molecular Weight: 316.27

I

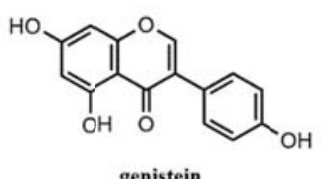

Chemical Formula: $\mathrm{C}_{15} \mathrm{H}_{10} \mathrm{O}_{5}$

Molecular Weight: 270.24

K

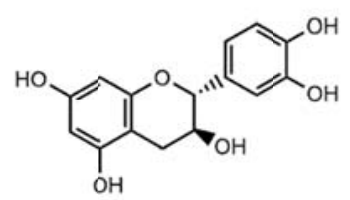

catechin

Chemical Formula: $\mathrm{C}_{15} \mathrm{H}_{14} \mathrm{O}_{6}$

Molecular Weight: 290.27
B<smiles>O=c1c(O)c(-c2ccc(O)c(O)c2)oc2cc(O)cc(O)c12</smiles>

Chemical Formula: $\mathrm{C}_{15} \mathrm{H}_{10} \mathrm{O}_{7}$

Molecular Weight: 302.24

D

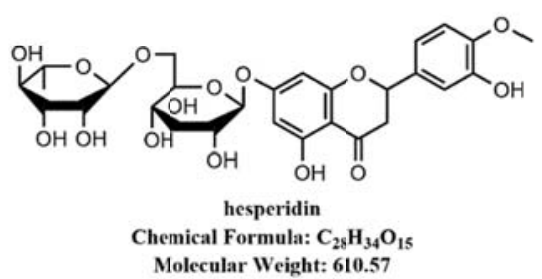

F

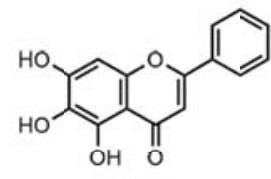

baicalein

Chemical Formula: $\mathrm{C}_{15} \mathrm{H}_{10} \mathrm{O}_{5}$

Molecular Weight: 270.24

$\mathrm{H}$

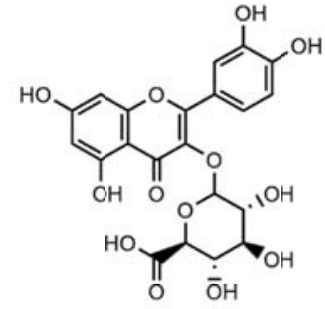

quercetin-3-O-glucuronide Chemical Formula: $\mathrm{C}_{21} \mathrm{H}_{18} \mathrm{O}_{13}$

Molecular Weight: $\mathbf{4 7 8 . 3 6}$

$\mathrm{J}$

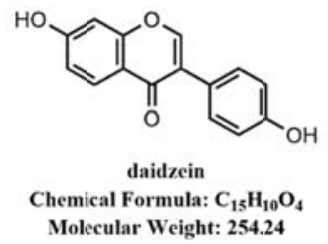


Figure 1. The chemical structures, molecular formulas and molecular weights of selected flavonoids. These flavonoids were selected because they were most commonly studied with significant cardiovascular effects. A: chrysin; B: quercetin; C: rutin; D: hesperidin; E: pinocembrin; F: baicalein; G: 3'-O-methyl-quercetin; H: quercetin-3-O-glucuronide; I: genistein; J: daidzein; and K: catechin.

\subsection{Phenolic acids}

Phenolic acids constitute another important class of polyphenols found in plants with well-established bioactivities. Both in vivo and in vitro, most of the phenolic acids showed antioxidative, anti-inflammatory and free radical scavenging activities, and could protect cells against injuries induced by various harmful factors.

Phenolic acids with varying chemical structures show different bioactivities. For example, phenolic acids isolated from a variety of legumes showed significant differences in phytochemical content and functional activities (Yao, et al. 2011). Protocatechuic acid (PA) is a naturally occurring phenolic acid found abundantly in $>500$ plants. It is structurally similar to other well-known antioxidants such as gallic acid, caffeic acid and vanillic acid. It exhibits different pharmacological effects such as its antioxidant activities, anti-inflammatory properties and interaction with several enzymes (Kakkar. \& Bais. 2014). Ferulic acid is a simple phenolic acid found in cereals. Spontaneously hypertensive rats (SHRs) treated with ferulic acid (50 $\mathrm{mg} \cdot \mathrm{kg}^{-1} \cdot \mathrm{d}^{-1}$ ) showed reduced systolic blood pressure, left ventricular diastolic stiffness, attenuated inflammatory cell infiltration, etc. (Alam, Sernia, \& Brown. 2013). In rats fed an atherogenic diet, veratric acid was found to significantly attenuate systolic and diastolic blood pressure and lipid peroxidation products after oral administration $(40 \mathrm{mg} / \mathrm{kg})$ for 30 days. In particular, it showed protective effects against atherogenic diet-induced hyperlipidaemia (Raja, Saravanakumar, \& Sathya, 2012). Salvianolic acids have been isolated from Salvia miltiorrhiza for use against cardiovascular diseases in traditional Chinese medicine for thousands of years (also known as Danshen) (Li, et al., 2007). To date, $>10$ different salvianolic acids have been identified: salvianolic acid A, B, C, D, E, F, G, etc. (Figure 2). Compared with the flavonoids in Danshen, salvianolic acids exhibit more potent antioxidant activity, among other bioactivities. Although these compounds directly scavenge free radicals, they may not be present at the high concentrations required in vivo. Their antioxidant activity may lead to the inhibition 
of pro-oxidant enzymes and the up-regulation of the expression of antioxidant enzymes, such as the activation of the Nrf2/haeme oxygenase-1 (HO-1) pathway (Pignatelli, et al., 2006; Zhang, et al., 2014). It is worth noting that polyphenols can also induce pro-oxidant responses in ECs leading to endothelial NO synthase activation as well as in cancer cells leading to apoptosis (Wang, et al., 2013). However, the pro-oxidant responses were concentration related. In addition, they showed tissue and cell specificity. This indicates that pro-oxidant responses can only be induced at a particular concentration in tissues and cells, which is very rare.

In general, the chemical structures of salvianolic acids possess the danshensu ( $(3$, 4-dihydroxyphenyl) lactic acid) unit, a simple monomeric compound isolated from Danshen. More precisely, these compounds are typically depsides of danshensu and caffeic acid derivatives with phenolic hydroxyl groups. 
A

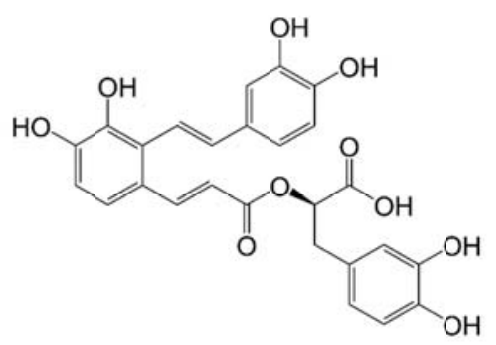

$$
\begin{gathered}
\text { salvianolic acid } \mathrm{A} \\
\text { Chemical Formula: } \mathrm{C}_{26} \mathrm{H}_{22} \mathrm{O}_{10} \\
\text { Molecular Weight: } 494.45
\end{gathered}
$$

C

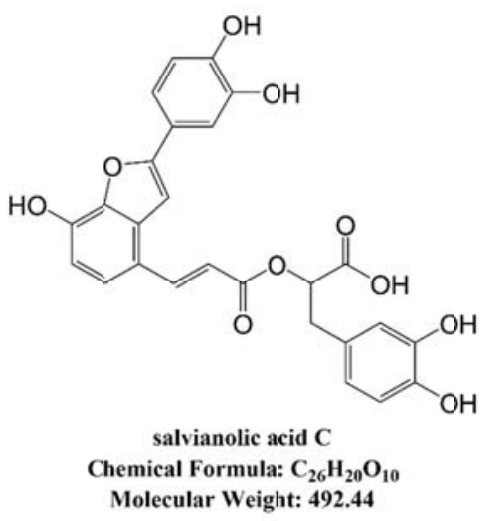

E

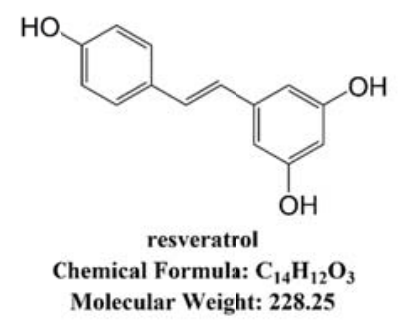

B

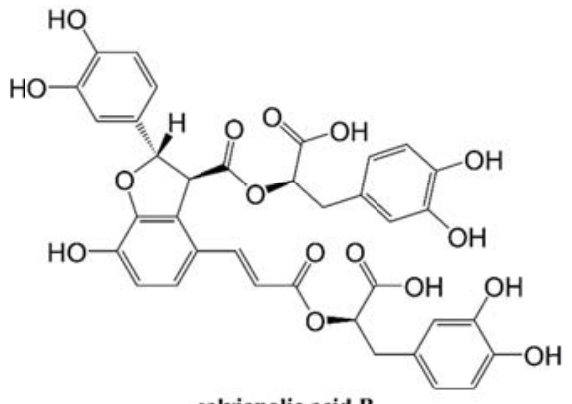

Chemical Formula: $\mathrm{C}_{36} \mathrm{H}_{30} \mathrm{O}_{16}$

Molecular Weight: $\mathbf{7 1 8 . 6 2}$

D<smiles>O=C(O)Cc1c(/C=C/C(=O)OC(Cc2ccc(O)c(O)c2)C(=O)O)ccc(O)c1O</smiles>
Chemical Formula: $\mathrm{C}_{20} \mathrm{H}_{18} \mathrm{O}_{10}$ Molecular Weight: $\mathbf{4 1 8 . 3 5}$

Figure 2. Chemical structures of phenolic acids and polyphenols. A: salvianolic acid A; B: salvianolic acid B; C: salvianolic acid C; D: salvianolic acid D; and E: resveratrol. 


\subsection{Others}

Various other polyphenol compounds, such as stilbenes, lignans and tannins, have been identified and studied for their therapeutic potential. These polyphenols have been found to have multiple cardiovascular effects. For example, resveratrol (Figure 2E) exhibits antioxidant and anti-inflammatory activities, prevent cell damage induced by oxidative stress, hypoxia and ischaemia (Carrizzo, et al., 2013; J. Li, et al., 2015; Lu, et al., 2010).

\section{Cardiovascular activities of polyphenols}

\subsection{Decrease in heart oxygen consumption or increase in oxygen supply}

The induction of coronary artery dilation is considered an important therapeutic strategy for IHD, as it restores the blood supply to the ischaemic area and minimizes the related damage.

Many studies have implicated nitric oxide (NO) in the modulation of $I / R$ injury (Bice, Jones, Chamberlain, \& Baxter, 2016). As a critical regulator of vascular tone, NO acts as a vasodilator by activating soluble guanylyl cyclase, subsequently increasing cyclic guanosine monophosphate (cGMP) in vascular smooth muscle cells. cGMP can be metabolized by cyclic nucleotide phosphodiesterases (PDEs). When the production and bioavailability of $\mathrm{NO}$ are adequate, vascular homeostasis is achieved. In myocardial ischaemia, the oxygen supply/demand ratio decreases while free oxygen radicals accumulate, thus reducing the bioavailability of NO. Because the $\mathrm{O}_{2}{ }^{-}$ radical is implicated in the destruction of $\mathrm{NO}$, it is important to establish whether $\mathrm{NO}$ may be made more bioavailable with increased antioxidant effects such as neutralizing the $\mathrm{O}_{2}{ }^{-}$radical and reducing its concentration. Hence, reduced levels of NO-destroying oxygen radicals can help in the dilation of coronary arteries.

Polyphenols extracted from grapes and wine (e.g., quercetin, tannic acid, resveratrol and malvidin) were found to increase coronary flow via endothelium-dependent relaxations (Fitzpatrick, Hirschfield, \& Coffey, 1993). Subsequent studies have shown that polyphenols lower the levels of NO-destroying oxygen radicals by scavenging these free radicals. In addition, polyphenols have been shown to promote NO production in ECs directly by inducing the overexpression of the endothelial nitric oxide synthase (eNOS) or by increasing eNOS activity. The 
pharmacological effects of polyphenols on myocardial ischaemia by either a decrease in oxygen demand or an increase in oxygen supply are summarized in Table 1. Polyphenols have also been found to scavenge free oxygen radicals and reduce oxidative stress by modulating glutathione (GSH) concentration, malondialdehyde (MDA) generation and the activities of pro-oxidant and antioxidant enzymes.

Salvianolic acid B (SalB) elevated the plasma NO levels via the nitrate reductase process, as observed in a rabbit heart I/R model, performed half an hour after ligation of the left anterior descending coronary artery (LADCA) and 1-4 hours for reperfusion (Yang, et al., 2008). In subsequent studies, SalB (40 mg/kg) was found to attenuate myocardial ischaemia injury in $\mathrm{C}_{57}$ mice and $\mathrm{eNOS}^{-/-}$mice by producing $\mathrm{NO}$ via eNOS phosphorylation, L-arginine uptake and cationic amino acid transporters expression (Pan, et al., 2011).

Resveratrol (3,5,4'-trihydroxy-trans-stilbene, Figure 2E), a plant-derived phenolic acid compound, increases the bioavailable NO content in SHRs by preventing NO and eNOS uncoupling. Resveratrol has been found to up-regulate eNOS activity by the following mechanisms: 1) The eNOS messenger RNA (mRNA) level is increased and mRNA stabilized in ECs (Wallerath, et al., 2002). 2) The eNOS phosphorylation ratio at the serine 1177 residue is increased to enhance eNOS activity by intensifying the interaction between oestrogen receptor $\alpha(E R \alpha)$ and Src-caveolin-1 at a concentration of $50 \mathrm{nM}$, which can be easily reached in vivo (Klinge, Wickramasinghe, Ivanova, \& Dougherty, 2008). 3) The intracellular levels of the endogenous eNOS inhibitor asymmetric dimethylarginine (ADMA) are decreased at concentrations ranging from 0.1 to $10 \mu \mathrm{M}$ (Frombaum, et al., 2011). 4) The eNOS activity is enhanced by inducing the SIRT1-mediated deacetylation of lysines 496 and 506 in the calmodulin-binding domain of eNOS (Mattagajasingh, et al., 2007).

Frombaum et al. indicated that piceatannol (4-[(E)-2-(3,5-dihydroxyphenyl) ethenyl] benzene-1,2-diol), as a resveratrol analogue, is more effective than resveratrol in restoring the expression and activity of the eNOS regulator dimethylarginine dimethylaminohydrolase in ECs under high-glucose oxidative stress.

Hydrolysable tannins, another group of phenolic acids isolated from the leaves of Rhus coriaria, show anti-ischaemic activity and endothelium-dependent vasorelaxant effects in isolated rabbit hearts and thoracic aortae at concentrations of 150-500 
$\mu \mathrm{g} / \mathrm{mL}$; the mechanisms included cyclooxygenase (COX) pathway activation, tumour necrosis factor alpha (TNF- $\alpha$ ) inhibition, eNOS activation and free radical and reactive oxygen species (ROS) scavenging (Beretta, Rossoni, Santagati, \& Facino, 2009).

Some flavonoids have been shown to induce endothelium-dependent vasodilation by increasing the bioavailability of NO. The flavonoid quercetin (2-(3,4-dihydroxyphenyl)-3,5,7-trihydroxy-4H-chromen-4-one) (Figure 1B) is most naturally prevalent, with reported endothelium-dependent vasodilatory effects via an increase in the bioavailability of NO. Perez-Vizcaino and Duarte reviewed the mechanisms of quercetin-induced NO production. Firstly, under conditions of enzymatically or chemically increased $\mathrm{O}_{2}^{-}$and thus accelerated NO metabolism, biomolecules such as quercetin, genistein, daidzein, biochanin A and ginkgo flavone aglycone will directly scavenge free oxygen radicals and therefore protect NO in cell-free systems or in ECs (López-López, et al., 2004). Secondly, flavonoids such as quercetin and monoacetyl-vitexin rhamnoside inhibit enzymatic sources of oxygen, such as xanthine oxidase and nicotinamide adenine dinucleotide phosphate (NADPH) oxidase (Busse, Kopp, \& Middleton, 1984). Thirdly, quercetin prevents BH4 oxidation and eNOS uncoupling (Romero, et al., 2009). Fourthly, under normal conditions, quercetin stimulates eNOS activity in a dose- and time-dependent manner following a sudden increase in intracellular $\mathrm{Ca}^{2+}$ concentrations, with a concomitant increase in NO production in ECs (Kuhlmann, et al., 2005). It is worth noting that quercetin is extensively metabolized into methylated and glucurono- and sulpho-conjugated forms that circulate in the plasma. Further, the glucurono-conjugates can be hydrolysed to the parent aglycone, which in turn accumulates in tissues and exerts vasodilatory and antihypertensive effects (Duarte \& Pérez-Vizcaíno, 2015).

Like resveratrol, most flavonoids up-regulate eNOS expression at both the mRNA and protein levels. This effect is mediated at least in part by SIRT1 and by hyperpolarization-dependent $\mathrm{Ca}^{2+}$ entry. Dimethylarginine dimethylaminohydrolase can regulate NO synthesis (Frombaum, et al., 2011; MacAllister, et al., 1996). In addition to NO, endothelium-dependent hyperpolarization can also lead to endothelium-dependent vasodilation. Quercetin is known to induce hyperpolarization-mediated endothelium-dependent relaxation and vasodilatation 
(Kuhlmann, et al., 2005). Furthermore, some flavonoids are potent inhibitors of PDE, which may contribute to their vasodilatory responses (Peluso, 2006; Ruckstuhl, Beretz, Anton, \& Landry, 1979).

Studies of human umbilical vein endothelial cells (HUVECs) have shown that 10 $\mu \mathrm{M}$ quercetin aglycone minimally reduces the eNOS level and significantly reduces the expression of ET-1, whereas human metabolites had no effect (Tribolo, et al., 2013). In human aortic ECs, 5 and $10 \mu \mathrm{M}$ quercetin and its metabolites (3'-O-methyl-quercetin and quercetin-3-O- glucuronide; Figure 1G, H) showed protective effects by activating adenosine monophosphate-activated protein kinase (AMPK) and inducing eNOS activity. These compounds caused an increase in S-nitrosothiol and nitrite concentrations (Shen, et al., 2012). Pretreatment of arteries with 5 and $10 \mu \mathrm{M}$ quercetin or its metabolites was found to prevent endothelium dysfunction in isolated C57BL mouse aortic rings (Shen, et al., 2012).

Catechin, a flavan-3-ol, is a naturally occurring flavonoid with antioxidant properties. $\quad$ Epigallocatechin gallate ((2R,3R)-5,7-dihydroxy-2-(3,4,5-trihydroxyphenyl)-3,4-dihydro-2H-1-benzopyran-3yl 3,4,5-trihydroxybenzoate, EGCG; Figure $1 \mathrm{~K}$ ) is a potent antioxidant and the most abundant catechin (water-soluble polyphenol) in tea. EGCG can modulate ROS production and eNOS activation, increase endothelium-derived NO levels via a PI3-kinase-dependent pathway in SHRs and enhance coronary flow (Potenza, et al., 2007). In rat aortic rings, EGCG has also been shown to cause endothelium-dependent vasodilation at concentrations of $1-50 \mu \mathrm{M}$ by activating eNOS via phosphatidylinositol 3-kinase-, protein kinase A (PKA)- and Akt-dependent pathways (Lorenz, et al., 2004). EGCG is also known to preserve the bioavailability of NO by reducing the levels of the endogenous NOS inhibitor ADMA (Tang, Hu, et al, 2006). Recent findings indicate that these effects are typical of the metabolites of EGCG. EGCG metabolites produced by intestinal bacteria also inhibit ACE (Takagaki \& Nanjo, 2015).

Some flavonoids have been shown to cause endothelium-dependent vasodilation that is highly dependent on PDE activity. Monoacetyl-vitexin rhamnoside, a flavonoid extracted from Crataegus species known to inhibit PDE, was suggested to improve myocardial perfusion in several isolated heart models (Schussler, Holzl, Rump, \& 
Fricke, 1995). API0134 from Andrographis paniculata improved cardiac function in dogs, decreased the extent of myocardial infarct size, alleviated the extent of myocardial injury and decreased the incidence of arrhythmias (Guo, Zhao, \& Fu, 1996). API0134 was also found to promote the production of prostaglandin, increase the prostacyclin I2 (PGI2) to thromboxane A2 (TXA2) ratio and inhibit the production of free radicals from granulocytes. API0134 also scavenges $\mathrm{H}_{2} \mathrm{O}_{2}, \mathrm{O}_{2}{ }^{-}$and $\mathrm{OH}$ significantly and inhibits the induced aggregation of washed human platelets. In addition, it significantly increases the NO and cGMP content and superoxide dismutase (SOD) activity, decreases endothelin and lipid hydroperoxide contents and maintains the NO/endothelin balance (Wang, Zhao, \& Xiang, 1997).

Phyto-oestrogens have been associated with decreased aortic stiffness, as measured by aortic pulse-wave velocity (Van der Schouw et al., 2002). Phyto-oestrogens such as genistein (Figure 1I) and daidzein (Figure 1, J) have been shown to relax pre-contracted coronary artery rings and mesenteric arterial rings of rabbit in a concentration-dependent manner. Like oestrogens, phyto-oestrogens relax arteries by significantly restoring the NO levels (Figtree, et al., 2000).

\subsection{Anti-atherosclerotic and cardioprotective activities}

Myocardial infarction therapy is more effective when atherosclerotic plaque formation is inhibited and the risk of thrombosis reduced. Restoration of endothelial function is an important approach, as the functional endothelium plays a critical role in anti-atherosclerotic and anti-thrombotic action. The cardiac endothelium is composed of endocardial ECs lining the heart chambers and vascular ECs lining the coronary microvasculature. Vascular EC-derived NO is a significant endogenous anti-atherosclerotic and anti-thrombotic molecule. It is known to inhibit the proliferation and migration of smooth muscle cells; enhance EC proliferation and migration; inhibit apoptosis; suppress platelet aggregation; and preventing platelet, leucocyte and monocyte adhesion to the endothelium. However, in myocardial ischaemia, the decrease in the oxygen supply/demand ratio leads to the accumulation of free oxygen radicals, which increases the risk of atherosclerotic plaque formation and thrombosis. As antioxidants, polyphenols enhance the bioavailability of NO or 
assist in the delivery of NO. Other anti-atherosclerotic and anti-thrombotic effects on blood cells and the vascular wall have also been studied.

Another study showed that the vasorelaxation effect reduced the incidence of coronary heart disease, the effect being mediated by the NO-cGMP pathway in some polyphenols (Fitzpatrick, et al; 1993). A recent study reported that anthocyanin supplementation could improve endothelium-dependent vasodilation by activating the NO-cGMP signalling pathway, enhancing the serum lipid profile and inhibiting inflammation (Zhu, Y, et al., 2011).

Resveratrol is widely used, as it prevents atherosclerosis via NO-dependent and NO-independent mechanisms. The NO-dependent mechanism of resveratrol is summarized as follows: First, resveratrol prevents superoxide production from NADPH oxidase and from the mitochondria. Second, resveratrol enhances the expression of several antioxidant enzymes including SOD isoforms, glutathione peroxidase 1 (GPx1), CAT and HO-1 (Li, Xia, \& Forstermann; 2012). Moreover, resveratrol is known to reverse eNOS uncoupling in hypercholesterolaemic ApoE-knockout (KO) mice by preventing BH4 oxidation and stimulating $\mathrm{BH} 4$ biosynthesis via upregulation of guanosine triphosphate (GTP) cyclohydrolase I (GPTCH1). Furthermore, resveratrol is also known to stimulate NO production by enhancing eNOS expression and activity via multiple mechanisms, including ER-mediated eNOS serine 1177 phosphorylation and SIRT1-mediated eNOS deacetylation. Polyphenols with similar structure to resveratrol are more likely to induce NO formation.

Resveratrol has also been proven to show anti-atherosclerotic and anti-thrombotic effects in a NO-independent manner by suppressing platelet adhesion, activation and aggregation, and in turn preventing thrombogenesis. Furthermore, resveratrol was shown to be a potent inhibitor of both nuclear factor kappa $B(N F-\kappa B)$ activation and NF-kB-dependent gene expression by inhibiting inhibitor of kappa B (IкB) kinase activity (Holmes-McNary \& Baldwin; 2000). Moreover, in silico docking results suggested that resveratrol forms stable complexes with platelet COX-1. Subsequent in vitro studies confirmed that resveratrol inhibits COX-1; modifies COX metabolite production to regulate platelet activation; and blocks the 
arachidonate-dependent synthesis of inflammatory agents such as thromboxane B2, hydroxyheptadecatrienoate and 12-hydroxyeicosatetraenoate (Crescente, et al., 2009).

Salvianolic acid A (SalA, Figure 1D) $(2.5-10 \mathrm{mg} / \mathrm{kg}$, i.v.) has been shown to significantly reduce thrombus weight in a murine model of an arteriovenous shunt. The anti-thrombotic effect may be related to its anti-platelet action and modulatory effect on haemorheology without affecting the coagulation system (Fan, et al., 2010). The anti-platelet and anti-thrombotic activities of SalA can be attributed to the inhibition of phosphoinositide 3-kinase (Huang, et al., 2010). Recently, SalA was shown to attenuate hypoxia-induced endothelial endoplasmic reticulum stress and apoptosis via down-regulation of very low-density lipoprotein (VLDL) receptor expression (Xie, Duan, Guo, Hu, \& Yu, 2015). It was also found to inhibit platelet-derived growth factor (PDGF)-BB-induced proliferation and migration of vascular smooth muscle cells, thus indicating the anti-atherogenic effects of SalA (Sun, et al., 2012).

SalB has been shown to exhibit anti-atherogenic effects in various studies. SalB prevented the oxidation of low-density lipoprotein (LDL) and inhibited lipid peroxidation by antagonizing the binding of oxLDL to CD36, in turn preventing the formation of foam cells (Karmin, et al., 2001). It also attenuated multiple inflammatory factors and inhibited the associated pathways including the expression of COX-2 (Chen, et al., 2006), matrix metalloproteinase (MMP)-2 and MMP-9 (Lin, et al., 2007) and TGF- $\beta /$ Smad (Wang, Tao, Yuan, Shen, \& Liu, 2010) or NF- $\kappa B$ signalling pathways (Wang, Hu, Gao, Guo, \& Fan, 2010), which may be involved in CD36-related pathways, thus leading to atherosclerotic and other vascular diseases. SalB (50 and $100 \mu \mathrm{M}$ ) inhibited PDGF-induced cell proliferation and migration, and markedly increased HO-1 expression via the reduction of reactive oxygen species and the NADP/NADPH ratio in vascular smooth muscle cells (Lee, MiRanSeo, \& Lee, 2014). In addition, SalB directly inhibited platelets isolated from Sprague-Dawley (SD) rat plasma in a dose-dependent manner (Li, et al., 2004). Wu et al. found that SalB inhibited platelet deposition, in a dose-dependent manner, from flowing, anti-coagulated whole blood to immobilized collagen at both venous and arterial shear rates by interfering with the collagen receptor $\alpha 2 \beta 1$ (Wu, et al., 2008). In a recent study, $\mathrm{Xu}$ et al. showed that SalB attenuated platelet-mediated inflammatory responses by inhibiting NF- $\mathrm{BB}$ activation in ECs even if the platelets were activated 
previously (Xu, et al., 2015).

Several molecular mechanisms have been proposed for the anti-platelet aggregation action of flavonoids: oxidative stress, protein tyrosine phosphorylation, calcium mobilization and NO pathway (Haouari \& Rosado, 2011). Gryglewski et al. were the first to attribute the anti-thrombotic effects of flavonols to their ability to scavenge superoxide (Gryglewski, 1987). They also attributed these effects to the inhibition of cyclic nucleotide PDEs and a subsequent increase in cAMP levels. Subsequently, the inhibitory effects of quercetin on protein kinase C (PKC)-dependent NADPH oxidase activation in platelets were established (Pignatelli, et al., 2006). In a recent study, reduced Fyn kinase activity as well as Syk and phospholipase C activation was implicated in the anti-aggregation effects of quercetin (Wright, et al., 2010). These effects were examined in vivo as well; however, Janssen et al. found no change in platelet aggregation, thromboxane B2 production, factor VII levels or other metrics when evaluating blood samples from 114 healthy volunteers receiving a dietary quercetin supplement (Janssen, et al., 1998).

Cocoa procyanidins stimulate the formation of a platelet aggregation inhibitor $\mathrm{PGI}_{2}$. These compounds also inhibit the formation of leukotrienes, which act as vasoconstrictors and stimulate inflammation (Manna, Mukhopadhyay, \& Aggarwal, 2000). Therefore, cocoa polyphenols inhibit coagulation and promote blood flow, thus preventing thrombosis (Csiszar, et al., 2008).

\subsection{Protective effect on myocardial cells and cardiac remodelling}

Ischaemia and $\mathrm{I} / \mathrm{R}$ can sometimes lead to cell death. It is worth noting that a delay in reperfusion can exacerbate ischaemic damage, as more ROS are produced when blood flow to the occluded artery is resumed. Thus, in principle, protecting cardiomyocytes from I/R-induced cell death would increase the viability of the myocardium, which is beneficial in both the short and long term after myocardial infarction. Therefore, cardiomyocyte apoptosis represents an excellent target in the treatment of myocardial infarction and I/R injury.

In recent studies, novel mechanisms of the myocardial protective action of polyphenols have been proposed: protection of mitochondrial function and the regulation of epigenetic modification-related enzyme activity. Moreover, polyphenols can also improve cardiac hypertrophy, ventricular remodelling and fibrosis after MI 
(Jiang, Chang, \& Dusting, 2010). In particular, polyphenols inhibiting cardiac remodelling or preventing myocardial apoptosis are summarized in Table 3.

In the 1990s, pharmacologists reported that SalA could protect the heart from I/R-induced damage both in vivo and in vitro (Du, Qiu, \& Zhang, 1995). Further research shows that SalA protects not only the heart in different animal models but also the vascular functions in normal and diabetic animal models when administrated orally or injected intravenously. SIRT1, a histone acetyltransferase, has been implicated in the regulation of hypoxia-induced and I/R-induced apoptosis in cardiomyocytes. Resveratrol has been proven to protect cells from apoptosis at low concentration by activating SIRT1. For example, in the cardiomyoblast cell line H9C2, $20 \mu \mathrm{M}$ resveratrol causes the rapid activation of SIRT1 and stimulates FoxO1 to induce cell cycle arrest, although it inhibits FoxO1-induced cell death. This indicates that resveratrol inhibits hypoxia-induced apoptosis in rat cardiac myoblasts (H9C2 cells) via the SIRT1-FoxO1 pathway (Chen, et al., 2009). Recently, resveratrol has been shown to up-regulate the expression of SIRT1 and to alleviate cardiac oxidative damage and left ventricular remodelling in an aged emphysema rat model. This indicates the therapeutic potential of resveratrol for cardiac injuries complicated by chronic obstructive pulmonary disease (COPD) (Hu, et al., 2013). More recently, resveratrol has been shown to reduce the ageing effect and alleviate hypoxiareperfusion (H/R) injury in cardiomyocytes during hypoxic preconditioning (HPC) depending on the regulation of SIRT1 (Zheng, Guo, Hong, Zheng, \& Wang, 2015). In addition, as an activator of SIRT1, resveratrol extends the lifespan of Caenorhabditis elegans (Morselli, et al., 2010) and protects the rat myocardium from I/R injury (Lekli, et al., 2010) via autophagy, as sirtuins have been proven to stimulate autophagy by deacetylation of autophagic proteins.

Mitochondrial, biochemical and morphological changes are typically observed in ischaemic cardiomyocytes, and these are also considered to be another cause of $I / R$ cardiomyocyte apoptosis. Resveratrol has gained interest in regulating mitochondrial dysfunction due to its effects on mitochondrial oxidative stress. In pigs with metabolic syndrome fed a high-cholesterol diet, resveratrol treatment lead to increased phosphorylation of mammalian target of rapamycin (p-mTOR) and decreased levels of p70 S6 kinase (P70S6K), lysosome-associated membrane protein (LAMP)-2 and autophagy-related gene 12-5 conjugates. These results suggested that resveratrol 
regulates autophagy signalling in chronic myocardial ischaemia (Sabe, Elmadhun, Dalal, Robich, \& Sellke, 2014).

Polyphenols also function via specific signalling pathways to reduce I/R-induced cardiomyocyte apoptosis. For example, resveratrol protects cardiomyocytes against anoxia/reoxygenation injury via the Toll-like receptor 4 (TLR4)/NF- $\mathrm{kB}$ signalling pathway. This indicates that the anti-apoptotic effect of resveratrol is associated with its anti-inflammatory effect (Zhang, et al., 2012). In addition, resveratrol exerts cardioprotective effects by activating adenosine receptors (ADRs) A1 and A3, regulating the downstream PI3K/Akt and cAMP response element-binding protein (CREB) pathway and controlling the expression and phosphorylation level of Bcl-2 (Das, Cordis, Maulik, \& Das, 2005). The activation of guanine nucleotide-binding protein-coupled receptors, such as ADR and opioid receptor (OPR), is known to repair I/R-induced damage in the heart. EGCG showed a cardioprotective effect by activating ADR, especially A (1) and A(2B) ADR, but not OPR in a Langendorff-perfused rat heart I/R model ( Lee, et al., 2012).

Magnolol (Figure 1E), a phenolic acid extracted from Magnolia officinalis, has been showed to have a protective effect on I/R-induced injury models. In 2008, Jin et al. reported the anti-apoptotic effect of magnolol in vivo via the extracellular signal-regulated kinase $1 / 2$ pathways in a rat I/R injury model (Jin, et al., 2008).

Caffeic acid (Figure 1F), a hydroxycinnamic acid found in fruits, grains and dietary supplements, is known to protect cardiac cells from isoproterenol-induced apoptosis related to cardiac enzymes, antioxidants and lipid peroxidation (Kumaran \& Prince, 2010).

$\alpha$-Mangostins (Figure 1G) is a polyphenolic xanthone extracted from the mangosteen fruit (Garcinia mangostana). $\alpha$-Mangostins can ameliorate isoproterenol-induced ultrastructural and functional abnormalities in the mitochondria, due to their ability to quench free radicals and activate the NO pathway (Sampath \& Kannan, 2009).

Insights into the effects of flavonoids and their mechanisms in myocardial cell protection have emerged in recent studies. Luteolin (3,4,5,7-tetrahydroxyflavone, Figure $2 \mathrm{G}$ ), belonging to the flavone subclass, has long been used in traditional 
Chinese medicine for the treatment of various diseases. Luteolin is known to reduce the myocardial infarct size, strengthen contractile function and attenuate cardiomyocyte apoptosis in both normal and diabetic rats with $\mathrm{I} / \mathrm{R}$ injury. The anti-apoptotic effect mediated by luteolin is associated with the regulation of the PI3K/Akt, ERK/PP1a/phospholamban (PLB)/sarco/endoplasmic reticulum $\mathrm{Ca}^{2+}$-ATPase (SERCA2a) and Janus kinase (JNK) signalling pathways, the up-regulation of the anti-apoptotic proteins fibroblast growth factor receptor 2 (FGFR2) and leukaemia inhibitory factor (LIF), increases in Bad phosphorylation and a decreased Bax-to-Bcl-2 ratio (D. Sun, et al., 2012).

Baicalein (5,6,7-trihydroxy-2-phenyl-4H-1-benzopyran-4-one, Figure $2 \mathrm{H}$ ) is one of the major flavonoids extracted from $S$. baicalensis. The biological activities of baicalein is partially due to its antioxidant effects. Baicalein mediates the opening of mitochondrial KATP and anion channels. Thus, pretreatment with baicalein at a concentration of $10 \mu \mathrm{M}$ offered significant protection against I/R injury and doxorubicin-induced cardiomyocyte apoptosis (Chang, et al., 2013). Song L et al. showed that baicalein reduced myocardial I/R injury by inhibiting 12/15 lipoxygenase activity; sequentially activating ERK1/2/AKT; and inhibiting p38 mitogen-activated protein kinase (MAPK), JNK1/2 and NF- $\mathrm{KB} / \mathrm{p} 65$ (Song, et al., 2014).

As a free radical scavenger, quercetin strengthens cardiac function and attenuates cardiomyocyte apoptosis. The elucidated mechanisms are as follows: inhibition of the JNK and c-fos cascade ( $\mathrm{Wu} \& \mathrm{Gu}, 2007)$; the up-regulation of phase 2 enzyme expression; and the regulation of ERK1/2, JNK, p38 and Akt phosphorylation and interleukin (IL)-1 $\beta$ (Gutierrez-Venegas \& Bando-Campos, 2010). Other flavonoids such as rutin, apigenin, myricetin, isoflavones, the phyto-oestrogen genistein and others have been found to exert an anti-apoptotic effect on cardiomyocytes by a direct antioxidant activity.

As a radical scavenger, EGCG is known to exert cardioprotective activity. Sheng $\mathrm{R}$ et al. reported the anti-apoptotic effect of EGCG on cardiomyocytes and its inhibitory effect on oxidative stress in cardiac hypertrophy induced by pressure overload (Sheng, Gu, Xie, Zhou, \& Guo, 2007). Later, they found that EGCG significantly inhibited $\mathrm{H}_{2} \mathrm{O}_{2}$-induced telomere attrition, telomeric repeat-binding factor 2 (TRF2) loss and p53 elevation in H9c2 cells. These results suggest that the anti-apoptotic effect of EGCG on cardiomyocytes is associated with a telomere-dependent apoptotic pathway (Sheng, Gu, Xie, Zhou, \& Guo, 2010). 


\subsection{Enhancing myocardial metabolism}

Excess fatty acids can impair the function of an ischaemic heart. In addition, fatty acid metabolism contributes to ischaemic heart injury. Approaches to enhance myocardial metabolism have been proposed. One involves increasing glucose uptake and decreasing the fatty acid levels. Another approach up-regulates glucose metabolism by increasing pyruvate dehydrogenase (PDH) levels (the rate-limiting enzyme for glucose oxidation). The latter strategy may lead to optimum energy metabolism in the heart and relieve symptoms of ischaemia (van der Schouw, et al., 2002). PA (Figure 1H) is a phenolic acid extracted from S. miltiorrhiza, with a structure typical of the class. PA was found to inhibit fatty acid oxidation and switch the energy substrate preference of the heart from fatty acids to glucose (Cao, et al., 2009). In addition, polyphenols were reported to prevent mitochondrial respiratory impairment perhaps via a decrease in oxidative stress and an increase in PGC- $1 \beta$ expression (Charles, et al., 2013). This may also help enhance myocardial metabolism.

\subsection{Pro-angiogenesis and cardiocyte regeneration}

Therapeutic angiogenesis is widely recognized as an effective strategy for reducing ischaemic injury by promoting the growth of new vessels or the maturation of pre-existing vessels. Some natural plant products have been reported to exert pro-angiogenic effects.

The pro-angiogenic effect of resveratrol has been observed primarily in myocardial infarction models both in vitro and in vivo (Kaga, Zhan, Matsumoto, \& Maulik, 2005) as follows: First, according to the human coronary arteriolar EC tube formation assay, resveratrol can significantly accelerate tubular morphogenesis, along with inducing HO-1 and vascular endothelial growth factor (VEGF) expression. Second, resveratrol increases thioredoxin (Trx)-1, HO-1 and VEGF expression in rat neonatal cardiomyocytes. Third, pretreatment with resveratrol $\left(1 \mathrm{mg} \cdot \mathrm{kg}^{-1} \cdot \mathrm{day}^{-1}\right)$ for 2 weeks reduces the infarct size in rats. Furthermore, the expression of Trx-1, HO-1 and VEGF increased after infarction in rats pretreated with resveratrol. In addition to the Trx-1-HO-1-VEGF pathway, several factors such as NO, NOS, NF- $\kappa$ B and specificity protein (SP)-1 are also attributed to the pro-angiogenic effects of resveratrol, because the expression of these factors is altered after infarction (Kaga, et 
al., 2005).

SMND-309, a novel metabolite of SalB, has been shown to enhance the migration, capillary-like structure formation and levels of VEGF, phosphorylated erythropoietin (EPO) receptor and phosphorylated STAT3 at doses of 3, 10 and 30 $\mu \mathrm{g} / \mathrm{mL}$. This indicates the significant angiogenic effect of SMND-309 on HUVECs. This is related to the up-regulation of VEGF via EPO receptor/STAT3 signalling pathways (Du, et al., 2013).

\section{Potential molecules for treatment of IHD}

Polyphenols have also been shown to retard vascular ageing and improve ageing-related endothelial dysfunction by inhibiting oxidative stress and inflammation, in turn reducing the incidence of myocardial ischaemia (Dal-Ros., et al., 2012; Idris Khodja, et al., 2012). Although polyphenols have multiple pharmacological effects beneficial against IHD, only a few will be developed for clinical practice. Both a high-purity single compound and a mixed natural product extract might help protect against IHD. Clinical studies are needed to compare the efficacy of the two types. Polyphenols represent an important source of drugs in the prevention and treatment of IHD.

\section{Conclusion and future perspective}

Natural products represent an important source of novel drugs. Accumulated data suggest the potential of many polyphenols in treating IHD by reducing myocardial oxygen consumption and/or increasing oxygen supply. Most polyphenols act by enhancing myocardial metabolism after $\mathrm{I} / \mathrm{R}$, inhibiting platelet aggregation and thrombosis, promoting angiogenesis or EC repair, protecting the remaining myocardial cells, restoring myocardial contraction, etc. Their beneficial effects and structural diversity make them potential candidates for the prevention and treatment of IHD. 
However, some issues need to be addressed. Firstly, their safety in humans must be carefully evaluated. Although natural products are generally considered to be less toxic, their potential toxicity, particularly of derivatives and analogues, should not be underestimated. Secondly, although polyphenols are adequately water soluble, their bioavailability must be improved. Finally, the phenolic hydroxyl groups present in these compounds, which contribute to their potent antioxidant activities, are easily oxidizable, thus raising the question of their stability in druggability. In conclusion, polyphenols constitute a valuable class of natural products with potentials for treating IHD.

Polyphenols are considered to be of great medicinal value due to their pleiotropic properties and structural diversity. In particular, they are good candidates for the prevention and treatment of IHD, as they reduce myocardial oxygen consumption and/or increase oxygen supply, enhance myocardial metabolism after I/R, inhibit platelet aggregation and thrombosis, promote angiogenesis or EC repair, protect the remaining myocardial cells or restore myocardial contraction.

The efficacy and safety of STA-2, the complex of natural polyphenols extracted from green tea, in the management of chronic stable angina have been evaluated in clinical phase II and II b trials. STA-2 significantly relieved chronic stable angina, as indicated by the change in total exercise time (TET) and time to $1 \mathrm{~mm}$ ST-segment depression during exercise tolerance testing (ETT). It also showed good safety for further development. Further, the State Food and Drug Administration (SFDA) of China has approved the use of new polyphenols-containing drugs, such as salvianolic acids for the treatment of chronic angina. At present, it is widely used in clinical practice due to its good efficacy and safety. The potential effects and underlying mechanisms of polyphenols are under study, to guide clinical practice. 


\section{Funding Sources}

This work was supported by the National Science Foundation (Grant number: 81102445) and by the PUMC Youth Fund and the Fundamental Research Funds for the Central Universities (Grant number: 33320140069). 
Table 1. The pharmacological effects of polyphenols on myocardial ischaemia via a decrease in oxygen demand or an increase in oxygen supply

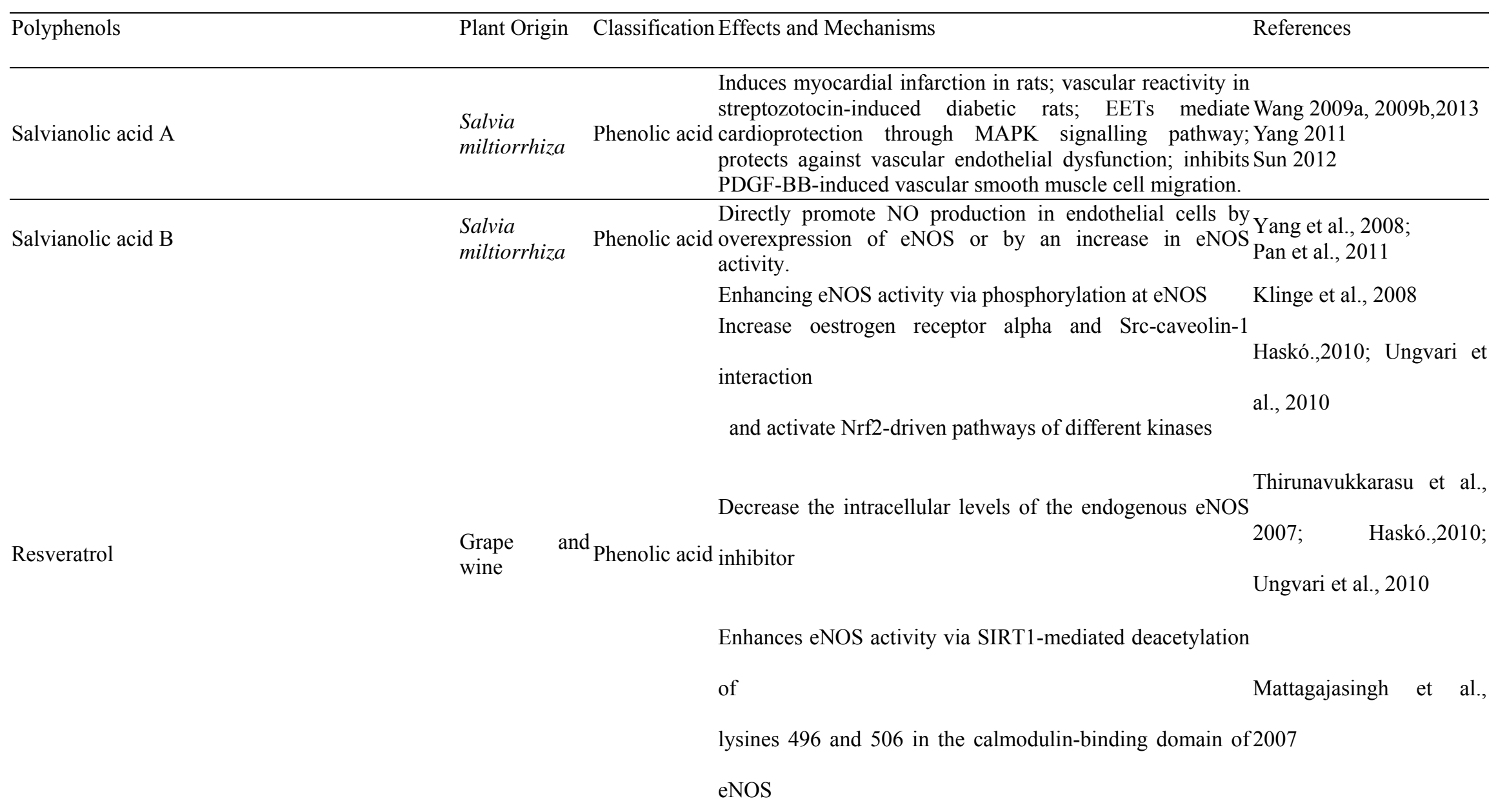




\section{Piceatannol}

Hydrolysable tannins

Curcumin

Quercetin

Quercetin,

3'-O-methyl-quercetin ,quercetin-3-O-glucuronide its
Restoring the eNOS regulator dimethylarginine

dimethylaminohydrolase,

stilbenoid eNOS expression and activity in endothelial cells underFrombaum et al.,2011

high-glucose

oxidative stress

Mediates endothelium-dependent vasorelaxant effect via

Rhus coriaria Phenolic acid COX pathway activation, TNF- $\alpha$ inhibition, eNOS Beretta et al., 2009

(Sumac) activation and free radical and ROS scavenging

Reduces cardiac preload in rabbits with pressure overload

and

ameliorate left ventricular function by inhibition of the Yao et al., 2004

remodelling

\section{Curcuma}

longa Linn. Curcumin

of the left ventricular collagen network associated with

suppression of myocardial TNF- $\alpha$ and MMP-2 expression

Induces isolated porcine coronary arteries relaxation

\section{through the}

Xu et al., 2007

action of NO, cGMP and adrenergic beta-receptor

Increases the NO bioavailability (by inhibiting oxygen Lopez-Lopez et al., enzymatic sources, i.e., xanthine oxidase and NADPH 2004 ; Busse et al., 1984; oxidase;

Romero

Avoids BH4 oxidation and eNOS uncoupling; Scavenges

Prevalen

Flavonoids

$\mathrm{O}_{2}^{-}$and thus protects $\mathrm{NO}$; Increases intracellular $\mathrm{Ca} 2$

concentrations, stimulates eNOS activity in a dose- and 2005 time-dependent manner),

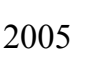

2005

Increases S-nitrosothiols and nitrite concentrations, activates 
metabolic

transformation

quercetin aglycone

Epigallocatechin gallate

Monoacetyl-vitexin rhamnoside (AVR)

API0134

AMPK and then induces eNOS activity in human aortic

endothelial cells

Causes slight reduction in eNOS and significantly reduces

ET-1

Tribolo et al., 2013

expression

Modulating ROS production and eNOS activation,

increasing

endothelium-derived NO via PI3 kinase-dependent Potenza et al.,2007

pathway,

enhancing coronary flow

Produces endothelium-dependent vasodilation at a

concentration of

Flavonoids $1-50 \mu \mathrm{M}$ by activating eNOS via a phosphatidylinositol Lorenz et al., 2004

3-kinase-, PKA- and Akt-dependent pathway in rat aortic

rings

Preserves bioavailability of NO by reducing the endogenous NOS inhibitor ADMA level

Crataegus

species

(Hawthorn,

Rosaceae)

Acanthaceae

plant

Andrographis Flavonoids

paniculata

(Buri. f.) Nees
Improves myocardial perfusion in isolated heart models

whosphodiesterase (PDE)-inhibitory properties

Schüssler et al.,1995

Promotes the production of prostaglandin; increases the PGI2/TXA2 ratio; prevents granulocytes from producing

free radicals;

scavenges $\mathrm{H}_{2} \mathrm{O}_{2}, \mathrm{O}^{2-}$ and $\cdot \mathrm{OH}$; and inhibits the induced Guo et al., 1996

aggregation

of washed human platelets

Increases NO and cGMP contents and SOD activity, Wang et al., 1997 
decreases

endothelin and lipid hydroperoxide contents and maintains

the

$\mathrm{NO} /$ endothelin balance 
Table 2. The polyphenols play inhibitory role in the platelet aggregation process.

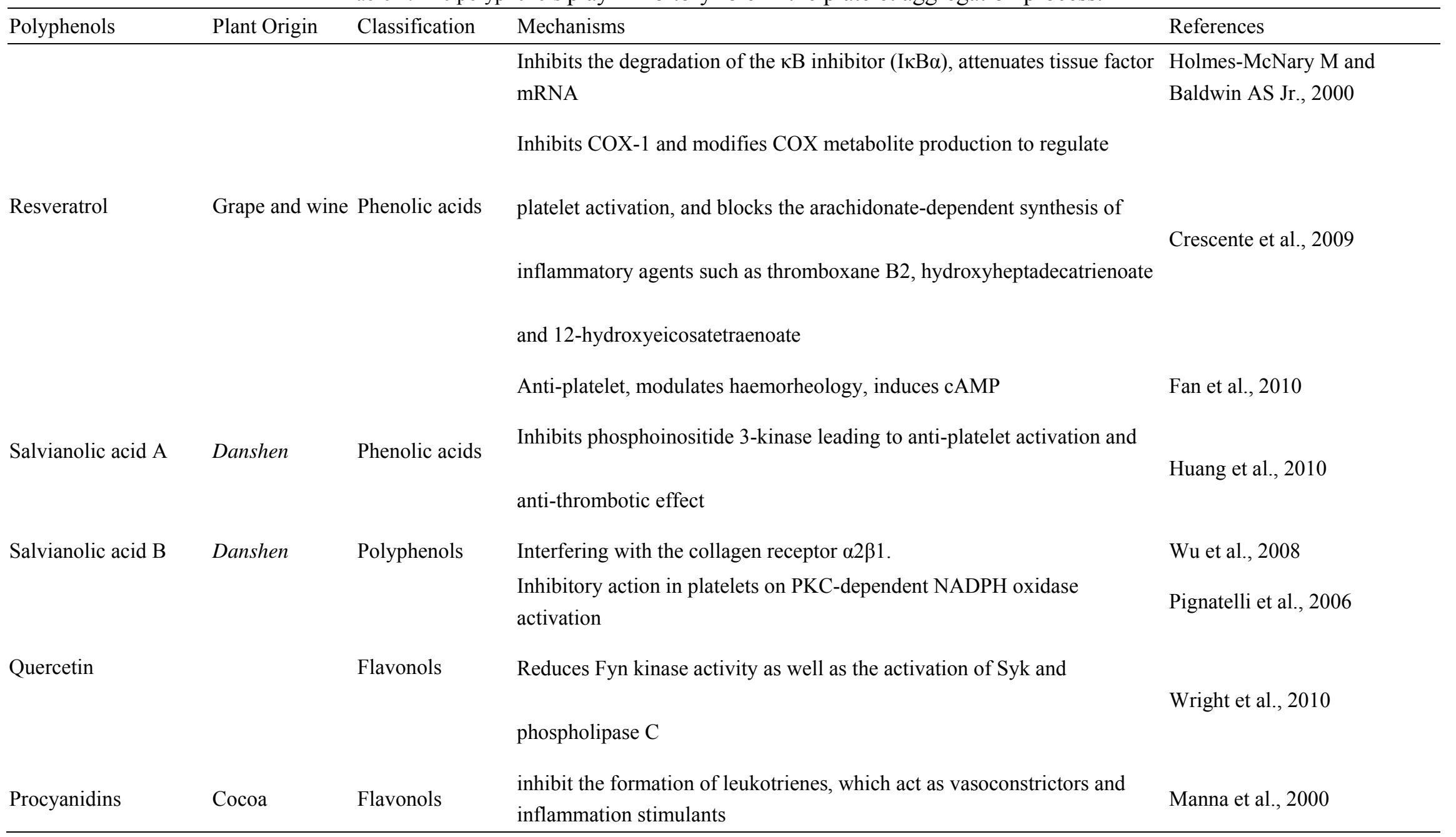



Table 3. Polyphenols inhibit cardiac remodelling or prevent myocardial apoptosis

\begin{tabular}{|c|c|c|c|c|}
\hline Polyphenols & Plant Origin & Classification & Effects and Mechanisms & References \\
\hline Resveratrol & Grape and wine & Phenolic acids & $\begin{array}{l}\text { Inhibits hypoxia-induced apoptosis in } \mathrm{H} 9 \mathrm{c} 2 \text { cells via the SIRT1-FoxO1 } \\
\text { pathway } \\
\text { Regulates autophagy signalling in chronic myocardial ischaemia; suppresses } \\
\text { increases in phosphorylated mammalian target of rapamycin (p-mTOR); and } \\
\text { decreases levels of p70 S6 kinase (P70S6K), lysosome-associated membrane } \\
\text { protein (LAMP)-2 and autophagy-related gene 12-5 conjugate }\end{array}$ & Chen et al., 2009 \\
\hline & & & $\begin{array}{l}\text { Protects cardiomyocytes against anoxia/reoxygenation injury via the } \\
\text { TLR4/NF-אB signalling pathway }\end{array}$ & Zhang et al., 2012 \\
\hline Magnolol & $\begin{array}{l}\text { Magnolia } \\
\text { officinalis }\end{array}$ & Phenolic acids & $\begin{array}{l}\text { Protects cardiomyocytes from ischaemia and reperfusion injury-induced } \\
\text { apoptosis via extracellular signal-regulated kinase } 1 / 2 \text { pathways in rat in vivo }\end{array}$ & Jin et al., 2008 \\
\hline Caffeic acid & $\begin{array}{l}\text { Fruits, grains and } \\
\text { dietary } \\
\text { supplements }\end{array}$ & Phenolic acids & $\begin{array}{l}\text { Ameliorates cardiac damage in ISO-induced myocardial infarction by } \\
\text { maintaining lipid peroxide metabolism due to its free radical scavenging and } \\
\text { antioxidant effects, and inhibiting cardiac cell apoptosis related to cardiac } \\
\text { enzymes, antioxidants and lipid peroxidation }\end{array}$ & $\begin{array}{l}\text { Kumaran KS and } \\
\text { Prince PS ., } 2010\end{array}$ \\
\hline Alpha-mangostins & Fruits & Phenolic acids & $\begin{array}{l}\text { Ameliorates isoproterenol-induced biochemical and morphological changes } \\
\text { in mitochondria, possibly mediated by the NO pathway and its ability to } \\
\text { quench free radicals }\end{array}$ & $\begin{array}{l}\text { Sampath PD and } \\
\text { Kannan V } 2009\end{array}$ \\
\hline Luteolin & & Flavonoids & $\begin{array}{l}\text { Regulating signalling pathways of PI3K/Akt, ERK/PP1a/PLB/SERCA2a and } \\
\text { JNK signalling pathways, up-regulating expression of anti-apoptotic proteins } \\
\text { FGFR2 and LIF, increasing BAD phosphorylation and decreasing the ratio } \\
\text { of Bax to Bcl-2, resulting in an anti-apoptotic effect }\end{array}$ & Sun et al., 2012; \\
\hline Baicalein & Scutellaria & Flavonoids & Protects against I/R injury and doxorubicin-induced cardiomyocyte & Chang et al., 2013 \\
\hline
\end{tabular}


baicalensis

\section{Quercetin}

\section{Epigallocatechin}

gallate apoptosis, by mediating the opening of mitochondrial KATP and anion channels

Inhibits 12/15 lipoxygenase, activates ERK1/2 /AKT, inhibits p38 MAPK,

JNK1/2, NF-kB/p65, reduces myocardial ischaemia/reperfusion injury

Song et al.,2014

Blocks JNK and c-fos cascade, and up-regulates phase 2 enzyme expression, Wu et al., 2007; and expression of ERK1/2, JNK, p38, AKT phosphorylation and IL-1 beta, Gutiérrez-Venegas gene

and Bando-Campos, 2010

Exerts anti-apoptotic effect on cardiomyocytes by inhibiting the effect of oxidative stress in pressure overload-induced cardiac hypertrophy
Sheng et al., 2007 
Figure Legends

Figure 1. The chemical structure, molecular formula and molecular weight of selected flavonoids. A: Chrysin; B: quercetin; C: rutin; D: hesperidin; E: pinocembrin; F: baicalein; G: 3'-O-methyl-quercetin; H: quercetin-3-O-glucuronide; I: genistein; J: daidzein; and K: catechins.

Figure 2. Chemical structures of phenolic acids and polyphenols. A: salvianolic acid A; B: salvianolic acid B; C: salvianolic acid C; D: salvianolic acid D; and E: resveratrol.

\section{References:}

Alam MA, Sernia C, Brown L. Ferulic acid improves cardiovascular and kidney structure and function in hypertensive rats. J Cardiovasc Pharmacol. 2013; 61(3): 240-249.

Beretta, G., Rossoni, G., Santagati, N. A., \& Facino, R. M. (2009). Anti-ischemic activity and endothelium-dependent vasorelaxant effect of hydrolysable tannins from the leaves of Rhus coriaria (Sumac) in isolated rabbit heart and thoracic aorta. Planta Medica, 75(14), 1482-1488.

Bhatt, S. R., Lokhandwala, M. F., \& Banday, A. A. (2011). Resveratrol prevents endothelial nitric oxide synthase uncoupling and attenuates development of hypertension in spontaneously hypertensive rats. European Journal of Pharmacology, 667(1-3), 258-264.

Bice, J. S., Jones, B. R., Chamberlain, G. R., \& Baxter, G. F. (2016). Nitric oxide treatments as adjuncts to reperfusion in acute myocardial infarction: a systematic review of experimental and clinical studies. Basic Research in Cardiology, 111(2), 1-15.

Bonetti, P. O., Holmes, D. R., Jr., Lerman, A., \& Barsness, G. W. (2003). Enhanced external counterpulsation for ischemic heart disease: what's behind the curtain? Journal of the American College of Cardiology, 41(11), 1918-1925.

Busse, W. W., Kopp, D. E., \& Middleton, E., Jr. (1984). Flavonoid modulation of human neutrophil function. Journal of Allergy and Clinical Immunology, 73(6), 801-809.

Cao, Y. G., Zhang, L., Ma, C., Chang, B. B., Chen, Y. C., Tang, Y. Q., Liu, X. D., \& Liu, X. Q. (2009). Metabolism of protocatechuic acid influences fatty acid oxidation in rat heart: new anti-angina mechanism implication. Biochemical Pharmacology, 77(6), 1096-1104.

Carrizzo, A., Forte, M., Damato, A., Trimarco, V., Salzano, F., Bartolo, M., Maciag, A., Puca, A. A., \& Vecchione, C. (2013). Antioxidant effects of resveratrol in cardiovascular, cerebral and metabolic diseases. Food and Chemical Toxicology, 61, 215-226.

Chang, W. T., Li, J., Vanden Hoek, M. S., Zhu, X., Li, C. Q., Huang, H. H., Hsu, C. W., Zhong, Q., Li, J., Chen, S. J., Vanden Hoek, T. L., \& Shao, Z. H. (2013). Baicalein preconditioning protects cardiomyocytes from ischemia-reperfusion injury via 
mitochondrial oxidant signaling. American Journal of Chinese Medicine, 41(2), 315-331.

Charles, A. L., Meyer, A., Dal-Ros, S., Auger, C., Keller, N., Ramamoorthy, T. G., Zoll, J., Metzger, D., Schini-Kerth, V., \& Geny, B. (2013). Polyphenols prevent ageing-related impairment in skeletal muscle mitochondrial function through decreased reactive oxygen species production. Experimental Physiology, 98(2), 536-545.

Chen, C. J., Yu, W., Fu, Y. C., Wang, X., Li, J. L., \& Wang, W. (2009). Resveratrol protects cardiomyocytes from hypoxia-induced apoptosis through the SIRT1-FoxO1 pathway. Biochemical and Biophysical Research Communications, 378(3), 389-393.

Chen, Y.-L., Hu, C.-S., Lin, F.-Y., Chen, Y.-H., Sheu, L.-M., Ku, H.-H., Shiao, M.-S., Chen, J.-W., $\&$ Lin, S.-J. (2006). Salvianolic acid B attenuates cyclooxygenase-2 expression in vitro in LPS-treated human aortic smooth muscle cells and in vivo in the apolipoprotein-E-deficient mouse aorta. Journal of Cellular Biochemistry, 98(3), 618-631.

Crescente, M., Jessen, G., Momi, S., Höltje, H.-D., Gresele, P., Cerletti, C., \& de Gaetano, G. (2009). Interactions of gallic acid, resveratrol, quercetin and aspirin at the platelet cyclooxygenase-1 level-Functional and modelling studies. Thrombosis and Haemostasis, 102(2), 336-346.

Csiszar, A., Labinskyy, N., Podlutsky, A., Kaminski, P. M., Wolin, M. S., Zhang, C., Mukhopadhyay, P., Pacher, P., Hu, F., de Cabo, R., Ballabh, P., \& Ungvari, Z. (2008). Vasoprotective effects of resveratrol and SIRT1: attenuation of cigarette smoke-induced oxidative stress and proinflammatory phenotypic alterations. American Journal of Physiology: Heart and Circulatory Physiology, 294(6), H2721-2735.

Das, S., Cordis, G. A., Maulik, N., \& Das, D. K. (2005). Pharmacological preconditioning with resveratrol: role of CREB-dependent Bcl-2 signaling via adenosine A3 receptor activation. American Journal of Physiology: Heart and Circulatory Physiology, 288(1), H328-335.

Dal-Ros, S., Bronner, C., Auger, C., \& Schini-Kerth, V. B. (2012). Red wine polyphenols improve an established aging-related endothelial dysfunction in the mesenteric artery of middle-aged rats: role of oxidative stress. Biochemical and Biophysical Research Communications, 419(2), 381-387.

Du, G., Zhu, H., Yu, P., Wang, H., He, J., Ye, L., Fu, F., Zhang, J., \& Tian, J. (2013). SMND-309 promotes angiogenesis in human umbilical vein endothelial cells through activating erythropoietin receptor/STAT3/VEGF pathways. European Journal of Pharmacology, 700(1-3), 173-180.

Du, G. H., Qiu, Y., \& Zhang, J. T. (1995). Protective effect of salvianolic acid a on ischemia-reperfusion induced injury in isolated rat heart. Yaо Хие Хие Bao. Acta Pharmaceutica Sinica, 30(10), 731-735.

Duarte, J., \& Pérez-Vizcaíno, F. (2015). Cardiovascular protection by flavonoids. Pharmacokinetic mystery. Ars Pharmaceutica (Internet), 56(4), 193-200.

El Haouari, M., \& A. Rosado, J. (2011). Modulation of Platelet Function and Signaling by Flavonoids. Mini Reviews in Medicinal Chemistry, 11(2), 131-142.

Estevinho, L., Pereira, A. P., Moreira, L., Dias, L. G., \& Pereira, E. (2008). Antioxidant and antimicrobial effects of phenolic compounds extracts of Northeast Portugal honey. Food 
and Chemical Toxicology, 46(12), 3774-3779.

Fan, H. Y., Fu, F. H., Yang, M. Y., Xu, H., Zhang, A. H., \& Liu, K. (2010). Antiplatelet and antithrombotic activities of salvianolic acid A. Thrombosis Research, 126(1), e17-e22.

Figtree, G. A., Griffiths, H., Lu, Y. Q., Webb, C. M., MacLeod, K., \& Collins, P. (2000). Plant-derived estrogens relax coronary arteries in vitro by a calcium antagonistic mechanism. Journal of the American College of Cardiology, 35(7), 1977-1985.

Fitzpatrick, D. F., Hirschfield, S. L., \& Coffey, R. G. (1993). Endothelium-dependent vasorelaxing activity of wine and other grape products. American Journal of Physiology-Heart and Circulatory Physiology, 265(2), H774-H778.

Frombaum, M., Therond, P., Djelidi, R., Beaudeux, J.-L., Bonnefont-Rousselot, D., \& Borderie, D. (2011). Piceatannol is more effective than resveratrol in restoring endothelial cell dimethylarginine dimethylaminohydrolase expression and activity after high-glucose oxidative stress. Free Radical Research, 45(3), 293-302.

Goszcz, K., Deakin, S. J., Duthie, G. G., Stewart, D., Leslie, S. J., \& Megson, I. L. (2015). Antioxidants in Cardiovascular Therapy: Panacea or False Hope? Front Cardiovasc Med, $2,29$.

Gryglewski, R. J., Korbut, R., Robak, J., \& Swies, J. (1987). On the mechanism of antithrombotic action of flavonoids. Biochemical Pharmacology, 36(3), 317-322.

Guo, Z., Zhao, H., \& Fu, L. (1996). Protective effects of API0134 on myocardial ischemia and reperfusion injury. Journal of Tongji Medical University, 16(4), 193-197.

Gutierrez-Venegas, G., \& Bando-Campos, C. G. (2010). The flavonoids luteolin and quercetagetin inhibit lipoteichoic acid actions on H9c2 cardiomyocytes. International Immunopharmacology, 10(9), 1003-1009.

Haskó G, Pacher P (2010). Endothelial Nrf2 activation: a new target for resveratrol? Am J Physiol Heart Circ Physiol 299:H10-12.

Hara, Y. (2011). Tea catechins and their applications as supplements and pharmaceutics. Pharmacological Research, 64(2), 100-104.

Harasym, J., \& Oledzki, R. (2014). Effect of fruit and vegetable antioxidants on total antioxidant capacity of blood plasma. Nutrition, 30(5), 511-517.

Holmes-McNary, M., \& Baldwin, A. S. (2000). Chemopreventive properties of trans-resveratrol are associated with inhibition of activation of the IкB kinase. Cancer Research, 60(13), 3477-3483.

Hu, Y. X., Cui, H., Fan, L., Pan, X. J., Wu, J. H., Shi, S. Z., Cui, S. Y., Wei, Z. M., \& Liu, L. (2013). Resveratrol attenuates left ventricular remodeling in old rats with COPD induced by cigarette smoke exposure and LPS instillation. Canadian Journal of Physiology and Pharmacology, 91(12), 1044-1054.

Huang, Z. S., Zeng, C. L., Zhu, L. J., Jiang, L., Li, N., \& Hu, H. (2010). Salvianolic acid A inhibits platelet activation and arterial thrombosis via inhibition of phosphoinositide 3-kinase. J. Thromb. Haemostasis, 8(6), 1383-1393.

Idris Khodja, N., Chataigneau, T., Auger, C., \& Schini-Kerth, V. B. (2012). Grape-derived polyphenols improve aging-related endothelial dysfunction in rat mesenteric artery: role of oxidative stress and the angiotensin system. PloS One, 7(2), e32039.

Iwashina, T. (2000). The Structure and Distribution of the Flavonoids in Plants. J. Plant Res., 
113(3), 287-299.

Janssen, K., Mensink, R. P., Cox, F. J., Harryvan, J. L., Hovenier, R., Hollman, P. C., \& Katan, M. B. (1998). Effects of the flavonoids quercetin and apigenin on hemostasis in healthy volunteers: results from an in vitro and a dietary supplement study. American Journal of Clinical Nutrition, 67(2), 255-262.

Jiang, F., Chang, C. W., \& Dusting, G. J. (2010). Cytoprotection by natural and synthetic polyphenols in the heart: novel mechanisms and perspectives. Current Pharmaceutical Design, 16(37), 4103-4112.

Jin, Y. C., Kim, K. J., Kim, Y. M., Ha, Y. M., Kim, H. J., Yun, U. J., Bae, K. H., Kim, Y. S., Kang, S. S., Seo, H. G., Lee, J. H., \& Chang, K. C. (2008). Anti-apoptotic effect of magnolol in myocardial ischemia and reperfusion injury requires extracellular signal-regulated kinase 1/2 pathways in rat in vivo. Experimental Biology and Medicine (Maywood, N.J.), 233(10), 1280-1288.

Kaga, S., Zhan, L., Matsumoto, M., \& Maulik, N. (2005). Resveratrol enhances neovascularization in the infarcted rat myocardium through the induction of thioredoxin-1, heme oxygenase-1 and vascular endothelial growth factor. Journal of Molecular and Cellular Cardiology, 39(5), 813-822.

Kakkar S, Bais S. A review on protocatechuic Acid and its pharmacological potential. ISRN Pharmacol. 2014: 952943.

Karmin, O., Lynn, E. G., Vazhappilly, R., Au-Yeung, K. K., Zhu, D.-Y., \& Siow, Y. L. (2001). Magnesium tanshinoate B (MTB) inhibits low density lipoprotein oxidation. Life Sciences, 68(8), 903-912.

Klinge, C. M., Wickramasinghe, N. S., Ivanova, M. M., \& Dougherty, S. M. (2008). Resveratrol stimulates nitric oxide production by increasing estrogen receptor $\alpha$-Src-caveolin-1 interaction and phosphorylation in human umbilical vein endothelial cells. The FASEB Journal, 22(7), 2185-2197.

Kuhlmann, C. R., Schaefer, C. A., Kosok, C., Abdallah, Y., Walther, S., Lüdders, D. W., Neumann, T., Tillmanns, H., Schäfer, C., \& Piper, H. M. (2005). Quercetin-induced induction of the NO/cGMP pathway depends on $\mathrm{Ca} 2+$-activated $\mathrm{K}+$ channel-induced hyperpolarization-mediated $\mathrm{Ca} 2+-$-entry into cultured human endothelial cells. Planta Medica, 71(6), 520-524.

Kumaran, K. S., \& Prince, P. S. (2010). Protective effect of caffeic acid on cardiac markers and lipid peroxide metabolism in cardiotoxic rats: an in vivo and in vitro study. Metabolism: Clinical and Experimental, 59(8), 1172-1180.

López-López, G., Moreno, L., Cogolludo, A., Galisteo, M., Ibarra, M., Duarte, J., Lodi, F., Tamargo, J., \& Perez-Vizcaino, F. (2004). Nitric oxide (NO) scavenging and NO protecting effects of quercetin and their biological significance in vascular smooth muscle. Molecular Pharmacology, 65(4), 851-859.

Lee, H. J., MiRanSeo, \& Lee, E. J. (2014). Salvianolic Acid B Inhibits Atherogenesis of Vascular Cells through Induction of Nrf2-dependent Heme Oxygenase-1. Current Medicinal Chemistry, 21(26), 3095-3106.

Lee, S. K., Kim, J. H., Kim, J. S., Jang, Y., Kim, J., Park, Y. H., Chun, K. J., \& Lee, M. Y. (2012). Polyphenol (-)-epigallocatechin gallate-induced cardioprotection may attenuate ischemia-reperfusion injury through adenosine receptor activation: a preliminary study. 
Korean Journal of Anesthesiology, 63(4), 340-345.

Lekli, I., Ray, D., Mukherjee, S., Gurusamy, N., Ahsan, M. K., Juhasz, B., Bak, I., Tosaki, A., Gherghiceanu, M., Popescu, L. M., \& Das, D. K. (2010). Co-ordinated autophagy with resveratrol and gamma-tocotrienol confers synergetic cardioprotection. Journal of Cellular and Molecular Medicine, 14(10), 2506-2518.

Li, H., Xia, N., \& Forstermann, U. (2012). Cardiovascular effects and molecular targets of resveratrol. Nitric Oxide, 26(2), 102-110.

Li, J., Xie, C., Zhuang, J., Li, H., Yao, Y., Shao, C., \& Wang, H. (2015). Resveratrol attenuates inflammation in the rat heart subjected to ischemia-reperfusion: Role of the TLR4/NF-kappaB signaling pathway. Mol Med Rep, 11(2), 1120-1126.

Li, L., Pang X.B., Chen B.N., Gao L., Wang L., Wang S.B., Wang S.B., Liu D.P., Du G.H.* (2013). Pinocembrin inhibits angiotensin II-induced vasoconstriction via suppression of the increase of $[\mathrm{Ca} 2+] \mathrm{i}$ and $\mathrm{ERK} 1 / 2$ activation through blocking AT1R in the rat aorta. Biochem Biophys Res Commun. 435(1): 69-75.

Li, M., Zhao, C., Wong, R. N. S., Goto, S., Wang, Z., \& Liao, F. (2004). Inhibition of shear-induced platelet aggregation in rat by tetramethylpyrazine and salvianolic acid B. Clinical Hemorheology and Microcirculation, 31(2), 97-103.

Li, X., Yu, C., Lu, Y., Gu, Y., Lu, J., Xu, W., Xuan, L., \& Wang, Y. (2007). Pharmacokinetics, tissue distribution, metabolism, and excretion of depside salts from Salvia miltiorrhiza in rats. Drug Metabolism and Disposition: The Biological Fate of Chemicals, 35(2), 234-239.Lin, S. J., Lee, I. T., Chen, Y. H., Lin, F. Y., Sheu, L. M., Ku, H. H., Shiao, M. S., Chen, J. W., \& Chen, Y. L. (2007). Salvianolic acid B attenuates MMP-2 and MMP-9 expression in vivo in apolipoprotein-E-deficient mouse aorta and in vitro in LPS-treated human aortic smooth muscle cells. Journal of Cellular Biochemistry, 100(2), 372-384.

Lorenz, M., Wessler, S., Follmann, E., Michaelis, W., Dusterhoft, T., Baumann, G., Stangl, K., \& Stangl, V. (2004). A constituent of green tea, epigallocatechin-3-gallate, activates endothelial nitric oxide synthase by a phosphatidylinositol-3-OH-kinase-, cAMP-dependent protein kinase-, and Akt-dependent pathway and leads to endothelial-dependent vasorelaxation. Journal of Biological Chemistry, 279(7), 6190-6195.

Lu, X., Ma, L., Ruan, L., Kong, Y., Mou, H., Zhang, Z., Wang, Z., Wang, J. M., \& Le, Y. (2010). Resveratrol differentially modulates inflammatory responses of microglia and astrocytes. Journal of Neuroinflammation, 7, 46.

MacAllister, R. J., Parry, H., Kimoto, M., Ogawa, T., Russell, R. J., Hodson, H., Whitley, G. S. J., \& Vallance, P. (1996). Regulation of nitric oxide synthesis by dimethylarginine dimethylaminohydrolase. British Journal of Pharmacology, 119(8), 1533-1540.

Manna, S. K., Mukhopadhyay, A., \& Aggarwal, B. B. (2000). Resveratrol suppresses TNF-induced activation of nuclear transcription factors NF-kappa B, activator protein-1, and apoptosis: potential role of reactive oxygen intermediates and lipid peroxidation. Journal of Immunology, 164(12), 6509-6519.

Mattagajasingh, I., Kim, C. S., Naqvi, A., Yamamori, T., Hoffman, T. A., Jung, S. B., DeRicco, J., Kasuno, K., \& Irani, K. (2007). SIRT1 promotes endothelium-dependent vascular relaxation by activating endothelial nitric oxide synthase. Proceedings of the National 
Academy of Sciences of the United States of America, 104(37), 14855-14860.

Morselli, E., Maiuri, M. C., Markaki, M., Megalou, E., Pasparaki, A., Palikaras, K., Criollo, A., Galluzzi, L., Malik, S. A., Vitale, I., Michaud, M., Madeo, F., Tavernarakis, N., \& Kroemer, G. (2010). Caloric restriction and resveratrol promote longevity through the Sirtuin-1-dependent induction of autophagy. Cell Death \& Disease, 1, e10.

Nicholson SK, Tucker GA, Brameld JM (2010). Physiological concentrations of dietary polyphenols regulate vascular endothelial cell expression of genes important in cardiovascular health. Br J Nutr 103:1398-1403.

Pan, C., Lou, L., Huo, Y., Singh, G., Chen, M., Zhang, D., Wu, A., Zhao, M., Wang, S., \& Li, J. (2011). Salvianolic acid B and tanshinone IIA attenuate myocardial ischemia injury in mice by NO production through multiple pathways. Therapeutic Advances in Cardiovascular Disease, 5, 99-111.

Peluso, M. R. (2006). Flavonoids attenuate cardiovascular disease, inhibit phosphodiesterase, and modulate lipid homeostasis in adipose tissue and liver. Experimental Biology and Medicine, 231(8), 1287-1299.

Pignatelli, P., Di Santo, S., Buchetti, B., Sanguigni, V., Brunelli, A., \& Violi, F. (2006). Polyphenols enhance platelet nitric oxide by inhibiting protein kinase C-dependent NADPH oxidase activation: effect on platelet recruitment. FASEB Journal, 20(8), 1082-1089.

Potenza, M. A., Marasciulo, F. L., Tarquinio, M., Tiravanti, E., Colantuono, G., Federici, A., Kim, J. A., Quon, M. J., \& Montagnani, M. (2007). EGCG, a green tea polyphenol, improves endothelial function and insulin sensitivity, reduces blood pressure, and protects against myocardial I/R injury in SHR. American Journal of Physiology: Endocrinology and Metabolism, 292(5), E1378-1387.

Qin TC, Chen L, Yu LX, Gu ZL (2001). Inhibitory effect of quercetin on cultured neonatal rat cardiomyocytes hypertrophy induced by angiotensin. Acta Pharmacol Sin 22:1103-1106.

Raj, P., Zieroth, S., \& Netticadan, T. (2015). An overview of the efficacy of resveratrol in the management of ischemic heart disease. Annals of the New York Academy of Sciences, 1348(1), 55-67.

Raja B, Saravanakumar M, Sathya G. Veratric acid ameliorates hyperlipidemia and oxidative stress in Wistar rats fed an atherogenic diet. Mol Cell Biochem. 2012;366(1-2):21-30.

Rasul, A., Millimouno, F. M., Ali Eltayb, W., Ali, M., Li, J., \& Li, X. (2013). Pinocembrin: a novel natural compound with versatile pharmacological and biological activities. Biomed Res Int, 2013, 379850.

Romero, M., Jimenez, R., Sanchez, M., Lopez-Sepulveda, R., Zarzuelo, M. J., O'Valle, F., Zarzuelo, A., Perez-Vizcaino, F., \& Duarte, J. (2009). Quercetin inhibits vascular superoxide production induced by endothelin-1: Role of NADPH oxidase, uncoupled eNOS and PKC. Atherosclerosis, 202(1), 58-67.

Ruckstuhl, M., Beretz, A., Anton, R., \& Landry, Y. (1979). Flavonoids are selective cyclic GMP phosphodiesterase inhibitors. Biochemical Pharmacology, 28(4), 535-538.

Sabe, A. A., Elmadhun, N. Y., Dalal, R. S., Robich, M. P., \& Sellke, F. W. (2014). Resveratrol regulates autophagy signaling in chronically ischemic myocardium. Journal of Thoracic and Cardiovascular Surgery, 147(2), 792-798; Discussion 798-799.

Santhakumar AB, Bulmer AC, Singh I (2014).A review of the mechanisms and effectiveness of 
dietary polyphenols in reducing oxidative stress and thrombotic risk. J Hum Nutr Diet 27:1-21.

Sampath, P. D., \& Kannan, V. (2009). Mitigation of mitochondrial dysfunction and regulation of eNOS expression during experimental myocardial necrosis by alpha-mangostin, a xanthonic derivative from Garcinia mangostana. Drug and Chemical Toxicology, 32(4), 344-352.

Schussler, M., Holzl, J., Rump, A. F., \& Fricke, U. (1995). Functional and antiischaemic effects of Monoacetyl-vitexinrhamnoside in different in vitro models. General Pharmacology, 26(7), 1565-1570.

Shen, Y., Croft, K. D., Hodgson, J. M., Kyle, R., Lee, I. L., Wang, Y., Stocker, R., \& Ward, N. C. (2012). Quercetin and its metabolites improve vessel function by inducing eNOS activity via phosphorylation of AMPK. Biochemical Pharmacology, 84(8), 1036-1044.

Sheng, R., Gu, Z. L., Xie, M. L., Zhou, W. X., \& Guo, C. Y. (2007). EGCG inhibits cardiomyocyte apoptosis in pressure overload-induced cardiac hypertrophy and protects cardiomyocytes from oxidative stress in rats. Acta Pharmacologica Sinica, 28(2), 191-201.

Sheng, R., Gu, Z. L., Xie, M. L., Zhou, W. X., \& Guo, C. Y. (2010). Epigallocatechin gallate protects $\mathrm{H} 9 \mathrm{c} 2$ cardiomyoblasts against hydrogen dioxides- induced apoptosis and telomere attrition. European Journal of Pharmacology, 641(2-3), 199-206.

Song, L., Yang, H., Wang, H. X., Tian, C., Liu, Y., Zeng, X. J., Gao, E., Kang, Y. M., Du, J., \& Li, H. H. (2014). Inhibition of 12/15 lipoxygenase by baicalein reduces myocardial ischemia/reperfusion injury via modulation of multiple signaling pathways. Apoptosis, 19(4), 567-580.

Sui, H., Chen, W. W., \& Wang, W. (2015). The key points of report on cardiovascular disease in China. Chinese Journal of Hypertension, 23(7), 627-629.

Sun, D., Huang, J., Zhang, Z., Gao, H., Li, J., Shen, M., Cao, F., \& Wang, H. (2012). Luteolin limits infarct size and improves cardiac function after myocardium ischemia/reperfusion injury in diabetic rats. PloS One, 7(3), e33491.

Sun, L., Zhao, R., Zhang, L., Zhang, T., Xin, W., Lan, X., Huang, C., \& Du, G. (2012). Salvianolic acid a inhibits PDGF-BB induced vascular smooth muscle cell migration and proliferation while does not constrain endothelial cell proliferation and nitric oxide biosynthesis. Molecules, 17(3), 3333-3347.

Takagaki, A., \& Nanjo, F. (2015). Effects of Metabolites Produced from (-)-Epigallocatechin Gallate by Rat Intestinal Bacteria on Angiotensin I-Converting Enzyme Activity and Blood Pressure in Spontaneously Hypertensive Rats. Journal of Agricultural and Food Chemistry, 63(37), 8262-8266.

Thirunavukkarasu M, Penumathsa SV, Koneru S, Juhasz B, Zhan L, Otani H et al. (2007). Resveratrol alleviates cardiac dysfunction in streptozotocin-induced diabetes: role of nitric oxide, thioredoxin, and heme oxygenase. Free Radic Biol Med 43, 720-729.

Tang, W. J., Hu, C. P., Chen, M. F., Deng, P. Y., \& Li, Y. J. (2006). Epigallocatechin gallate preserves endothelial function by reducing the endogenous nitric oxide synthase inhibitor level. Canadian Journal of Physiology and Pharmacology, 84(2), 163-171.

Tribolo, S., Lodi, F., Winterbone, M. S., Saha, S., Needs, P. W., Hughes, D. A., \& Kroon, P. A. (2013). Human metabolic transformation of quercetin blocks its capacity to decrease 
endothelial nitric oxide synthase (eNOS) expression and endothelin-1 secretion by human endothelial cells. Journal of Agricultural and Food Chemistry, 61(36), 8589-8596.

Ueno M, Kodali M, Tello-Montoliu A, Angiolillo DJ (2011). Role of platelets and antiplatelet therapy in cardiovascular disease. J Atheroscler Thromb 18(6):431-442.

Ungvari Z, Bagi Z, Feher A, Recchia FA, Sonntag WE, Pearson K et al. (2010) Resveratrol confers endothelial protection via activation of the antioxidant transcription factor Nrf2. Am J Physiol Heart Circ Physiol 299:H18-H24.

van der Schouw, Y. T., Pijpe, A., Lebrun, C. E., Bots, M. L., Peeters, P. H., van Staveren, W. A., Lamberts, S. W., \& Grobbee, D. E. (2002). Higher usual dietary intake of phytoestrogens is associated with lower aortic stiffness in postmenopausal women. Arteriosclerosis, Thrombosis, and Vascular Biology, 22(8), 1316-1322.

Wallerath, T., Deckert, G., Ternes, T., Anderson, H., Li, H., Witte, K., \& Forstermann, U. (2002). Resveratrol, a polyphenolic phytoalexin present in red wine, enhances expression and activity of endothelial nitric oxide synthase. Circulation, 106(13), 1652-1658.

Wang SB, Tian S, Yang F, Yang HG, Yang XY, Du GH. Cardioprotective effect of salvianolic acid A on isoproterenol-induced myocardial infarction in rats. Eur J Pharmacol, 2009a, 615(1-3): 125-132.

Wang SB, Yang XY, Tian S, Yang HG, Du GH. Effect of salvianolic acid A on vascular reactivity of streptozotocin-induced diabetic rats. Life Sci, 2009b, 85(13-14): 499-504.

Wang SB, Zhang WK, Pang XB, Li L, He GR, Yang XY, Fang LH, Zhang JT, Du GH. EETs mediate cardioprotection of salvianolic acids through MAPK signaling pathway. Acta Pharmaceutica Sinica B, 2013,3(1):25-31.

Wang, HW, Zhao, HY, \& Xiang, SQ. (1997). Effects of Andrographis Paniculata Component on Nitric Oxide, Endothelin and Lipid Peroxidation in Experimental Atheroscierotic Rabbits [J]. Chinese Journal of Integrated Traditional and Western Medicine, 17(9), 547-549.

Wang, Q.-L., Tao, Y.-Y., Yuan, J.-L., Shen, L., \& Liu, C.-H. (2010). Salvianolic Acid B prevents epithelial-to-mesenchymal transition through the TGF- $\beta 1$ signal transduction pathway in vivo and in vitro. BMC Cell Biology, 11, No pp given.

Wang, S. X., Hu, L. M., Gao, X. M., Guo, H., \& Fan, G. W. (2010). Anti-inflammatory activity of salvianolic acid B in microglia contributes to its neuroprotective effect. Neurochemical Research, 35(7), 1029-1037.

Wang, Z.-s., Luo, P., Dai, S.-h., Liu, Z.-b., Zheng, X.-r., \& Chen, T. (2013). Salvianolic Acid B Induces Apoptosis in Human Glioma U87 Cells Through p38-Mediated ROS Generation. Cellular and Molecular Neurobiology, 33(7), 921-928.

Wright, B., Moraes, L. A., Kemp, C. F., Mullen, W., Crozier, A., Lovegrove, J. A., \& Gibbins, J. M. (2010). A structural basis for the inhibition of collagen-stimulated platelet function by quercetin and structurally related flavonoids. British Journal of Pharmacology, 159(6), 1312-1325.

Wu, Y., \& Gu, Y. M. (2007). Effect of EGb and quercetin on culture neonatal rat cardiomyocytes hypertrophy and mechanism. Chinese journal of applied physiology, 23(2), 138-142.

Wu, Y. P., Zhao, X. M., Pan, S. D., Guo, D. A., Wei, R., Han, J. J., Kainoh, M., Xia, Z. L., de Groot, P. G., \& Lisman, T. (2008). Salvianolic Acid B inhibits platelet adhesion under 
conditions of flow by a mechanism involving the collagen receptor $\alpha 2 \beta 1$. Thrombosis Research, 123(2), 298-305.

Xie, P., Duan, Y., Guo, X., Hu, L., \& Yu, M. (2015). SalA attenuates hypoxia-induced endothelial endoplasmic reticulum stress and apoptosis via down-regulation of VLDL receptor expression. Cellular Physiology and Biochemistry, 35(1), 17-28.

Xin, W.Y., Tian, S., Song, J.K., He, G.R., Mu, X., Qin, X.M., \& Du, G.H. (2014). Research progress on pharmacological actions and mechanism of baicalein and baicalin. Current Opinion Complement Alternat Med, 1(2), e00010.

Xu, S., Zhong, A., Bu, X., Ma, H., Li, W., Xu, X., \& Zhang, J. (2015). Salvianolic acid B inhibits platelets-mediated inflammatory response in vascular endothelial cells. Thrombosis Research, 135(1), 137-145.

Xu PH, Long Y, Dai F, Liu ZL (2007). The relaxant effect of curcumin on porcine coronary arterial ring segments. Vascul Pharmacol 47:25-30.

Yao QH, Wang DQ, Cui CC, Yuan ZY, Chen SB, Yao XW et al. (2004). Curcumin ameliorates left ventricular function in rabbits with pressure overload: inhibition of the remodeling of the left ventricular collagen network associated with suppression of myocardial tumor necrosis factor-alpha and matrix metalloproteinase-2 expression. Biol Pharm Bull 27:198-202.

Yang, F. G., Zhang, A. Y., Chen, Z. Y., Lian, Z. X., Liu, G. X., \& Dong, G. X. (2008). Effects of salvianolic acid $\mathrm{B}$ on cardiovascular endothelial cells and platelet activation in a rabbit model of ischemia-reperfusion. Zhong Xi Yi Jie He Xue Bao. Journal of Chinese Integrative Medicine, 6(12), 1250-1254.

Yao Y, Cheng X, Wang L, Wang S, Ren G. Biological potential of sixteen legumes in China. Int J Mol Sci. 2011;12(10):7048-58.

Zhang, C., Lin, G., Wan, W., Li, X., Zeng, B., Yang, B., \& Huang, C. (2012). Resveratrol, a polyphenol phytoalexin, protects cardiomyocytes against anoxia/reoxygenation injury via the TLR4/NF-kappaB signaling pathway. International Journal of Molecular Medicine, 29(4), 557-563.

Zhang, H., Liu, Y. Y., Jiang, Q., Li, K. R., Zhao, Y. X., Cao, C., \& Yao, J. (2014). Salvianolic acid A protects RPE cells against oxidative stress through activation of Nrf2/HO-1 signaling. Free Radical Biol. Med., 69(1), 219-228.

Zheng, H., Guo, H., Hong, Y., Zheng, F., \& Wang, J. (2015). The effects of age and resveratrol on the hypoxic preconditioning protection against hypoxia-reperfusion injury: studies in rat hearts and human cardiomyocytes. European Journal of Cardio-Thoracic Surgery, 48(3), 375-381. 